Chemical Physics 57 (1981) 29-44

North-Holland Publishing Company

\title{
DYNAMICS OF ENERGY TRANSPORT IN TERNARY MOLECULAR SOLIDS. I. NAPHTHALENE STEADY STATE FLUORESCENCE $ं$
}

\author{
Panos ARGYRAKIS* and Raoul KOPELMAN \\ Department of Chemistry, The Unitersity of Michigan, Ann Arbor, Michigan 48109, USA
}

Received 19 September 1980

Revised manuscript received 15 December 1980

\begin{abstract}
Energy transport experiments were conducted at liquid helium temperatures on ternary single crystals (host/guest/ supertrap, i.e. $\mathrm{C}_{10} \mathrm{H}_{8} / \mathrm{C}_{10} \mathrm{D}_{8} /$ betamethylnaphthalene- $d_{10}$ ) at guest concentrations of $50-99 \%$ and very low (about $10^{-5}$ ) supertrap concentrations. The relative supertrap to guest steady-state funorescence gives a measure of the exciton percolation probability (migration to the supertrap). The onset of efficient transport is observed at about $85 \% \mathrm{C}_{10} \mathrm{H}_{8}$, compared to about $50 \%$ at higher supertrap concentrations bearing out the kinetic nature of the transport (e.g. dynamic percolation). The wealth of data are well fitted by an efiectively two-dinensional percolation formalism containing a single parameter of "coherency", i.e. an exciton mean free path (in nearest neighbor lattice units), extrapolated to the pure crystal (naphthalene). Our result gives about $10^{2}$ or more correlated hoppings (retaining directional memory) and is consistent with linewidth information and with time-resolved studies.
\end{abstract}

\section{Introduction}

The topic of energy transport in disordered systerns has certainly attracted a great deal of attention during the last decade [1-7], but especially during the last year or two [8-30]. Many new theoretical and experimental approaches have been reported. In all this work it is now accepted that the system of interest is made of donor sites and lower energy acceptor sites (supertrap). In more refined studies a third component is introduced with still higher energy: the host (while the donor is the guest).

Most of the above mentioned theoretical approaches do not take into consideration the local heterogeneity of the disordered systems, which is of primary importance even for randomly mixed crystals with two or more components. In a substitutionally random mixed crystal each component forms several sets of groups or clusters whose size, shape, dimen-

† Supported by NIH Grant 2R01 NS08116-10AI.

- Present addiess: Department of Physics, University of Crete, Heraklion, Crete, Greece. sionality, etc. will depend upon the concentration of this particular component in the crystal. There is good evidence $[31-38,43]$ to suggest that formalisms which do recognize these clusterization effects are advantageous for the description of transport properties and mechanisms. Percolation theory was applied to molecular aggregates $[2,31]$ only recently. In this approach it is usually necessary to first derive the cluster distribution for a binary system via a Monte Carlo simulation technique. This gives the size and frequency distribution of clusters as a function of topology, extent of interactions, and the binary concentration. The special algorithm [42] that made these calculations possible within a reasonable amount of computer time and memory space opened the way to new methods, as for example the accurate determination of critical exponents in critical phenomena [44], and details of energy migration in excited states [32]. For the latter situation, which is the focus of this paper, it is advantageous to consider a temary system, where the concentration of the third component 
is considerably lower then the other two, a physically reasonable situation when this component serves in the role of impurity trap (supertrap sensor, acceptor) molecules. Theoretically this was done by deriving an analytical sorm incorporating the results of simulations on binary lattices, rather than by inserting a third species into the computer simulated lartice. For the ternary systems in this study we identify the three species as host, guest, and supertrap lattice sites. This sequence is one of decreasing energy for the first excited state (singlet or triplet).

The guest species is the part of the lattice where excitonic energy transfer is taking place and is being monitored. Obviously, oniy at high guest concentration is energy transfer possible. The presence of the higher energy host (inaccessible to energy transfer) overshadows all scattering contributions from defects, impurities, surfaces, etc; also variation of its concentration results in varying the amount of scattering in an essentially controlled manner. The supertrap is still higher in concentration than any other impurity or X-trap. Thus, all interference by trapping from unaccounted species is neutralized. It aiso serves the purpose of sensing and registering all excitonic energy that is trapped by it.

We chose the well known naphthalene system for our experimental investigation, where two isotopic species serve as host and guest $\left(\mathrm{C}_{10} \mathrm{D}_{8}\right.$ and $C_{10} \mathrm{H}_{8}^{\mathrm{r}}$, respectively), and a slightiy different molecule. beta-methylnaphthalene (BMN), is the supertrap species. This ternary system has been well studied for both singlet and triplet exciton transport $[33-35,43]$. We report here on our steady-state study on this system. Time resolved studies are described in part II of this series.

In a recent series of papers information has become available [36-38] on the mode of propagation of a rardom walker on random lattices. This includes the long range correlation of random walks, hitherto called "coherence" $l$. We present here our model of transport for a mixed three-component system which is based on the consideration of random walks on the largest percolating cluster made of guest sites. We also show results of experiments designed to test our transport model, and we treat "coherence" as an adjustable parameter. Much has been recently written [5-7] on ways of estimating coherence, including the experimental observation of the exciton-phonon coupling by measuring the zero-phonon linewidth [5-7]. However, the interpretation is quite ambiguous (e.g., due to the inhomogeneous linebroadening. the experiment will provide only an upper limit to l). We present here a somewhat different approach to this problem.

In section 2 we describe the experimental apparatus used together with the technique of crystal growing and establishing the crystal concentrations. In section 3 we develop the formalism based on percolation considerations and show how it is applicable to our experiments. In section 4 we discuss temperature effects, and innally in section 5 we discuss the validity of our results and conclusions.

\section{Experimental}

Several mixed naphthalene crystals were grown using chemicals from Materials Limited $\left(\mathrm{C}_{10} \mathrm{H}_{8}\right.$, specified at $99.999 \%$ pure $)$, and from Thompson-Packard and Merck, Sharp and Dohme $\left(C_{10} D_{3}\right.$, specified at 99 atom \% D). All materials were zone refined. The main impurity in these crystals is beta-methylnaphthalene, $B M N$, which was present only in the $C_{10} D_{8}$ (in the deuterated form) but not (detectably) in the $\mathrm{C}_{30} \mathrm{H}_{8}$ which was chemically more pure. The crystals were grown using the Bridgman technique. The chemicals were mixed in clean glass tubes, where they were subjected to several cycles of melting and degasing to assure that all foreign gases were removed before the tubes were sealed under high vacuum. After thorough mixing to achieve homogeneity they remained 2-3 days in the crystal grower where the moving mechanism was stopped at critical stages to better anneal the crystals. Finally, the sealed tubes were broken off, the crystals cleaved along the $a b$ plane, mounted on a strain-free 
steel sample holder, and immersed in the cryostat.

The apparatus used for all experiments is shown in fig. 1. The sample is in the cryostat(CRYO) at point $S$, in liquid helium bath for the 4.2 and $1.8 \mathrm{~K}$ studies, or in liquid nitrogen for the $77 \mathrm{~K}$ studies. The source of light was a high pressure Xe lamp (1600 or $2300 \mathrm{~W}$ Hanovia). Radiation was properly filtered [39] before focusing on the crystal with lens systems L. L1, and L2. The spectrometer is a CzernyTurner double, $1 \mathrm{~m}$, scanning Jarrell-Ash, model number 25-100, with a resolution of $1 \mathrm{~cm}^{-1}$ (first order, at $3000 \AA$ ). The detection of the dispersed signal is via an ITT F4013 tube at position PMT1. The signal monitoring is done with an SSR photon counter, model 1110. A Westinghouse Fe-Ne hollow cathode lamp is used for calibration, in position FE via a rotating chopper (position $\mathrm{CH}$ ). The signal is then digitized using a home-made analog-to-digital converter [40], and fed to a Kennedy magnetic tape recorder, at TR 1 , where it is recortied for permanent storage. A new, revised, and extended software package [41] is used for the analysis of data that includes operations such as



Fig. 1. Instrumenta! block diagram. SSR - photon counting amplifier and discriminator, PMT 1 - ITT F4103 photomultiplier, $\mathrm{CH}$ - PAR model 125 chopper, JA 25-100 - Jarrell-Ash $1 \mathrm{~m}$ double Czemy-Tumer spectrometer, PMT2 - RCA 1P28 photomultiplier, SSR 1110 - digital synchronous computer and photon counter, TRI - tape recorder interface, $T$ - Kennedy nodel 1600 incremental tape recorder, R - Fiouston Instruments Omniscribe dial-pen recorder, TRIG - trigger circuit, A/D analog/digital converter for PAR 162, PAR 162 - boxcar averager, FE - iron hollow cathode calibraticn lamp, SCAN Molectron DL 400 scan control, TEK 7904 - Tektronix $500 \mathrm{MHz}$ oscilloscope. $X$ - high pressure Xe arc lamps (1600 W), $\mathrm{Ar}^{+}$- Spectra Physics model $54 \mathrm{Ar}$ ion laser, M, BS, L - various mirrors, lenses and beam steerers, CRYO - liquid helium immersion cryostat, HN - Spectra Physics He-Ne alignment laser, DL 400 - Molectron pulsed dye laser, UV 1000 - Molectron nitrogen laser, Table -5 ton steel-plated concrete table. 
smouthing, despiking, condensing, calibrating, concatinating, integrating, etc.

The sample concentrations were estimated from the weights of the materials mixed. The BMN impurity, due to its low concentration, is a little more difficult to estimate. Mass spectra were used to verify the $\mathrm{C}_{10} \mathrm{H}_{8} / \mathrm{C}_{10} \mathrm{D}_{8}$ ratio, and absorption spectroscopy was used to establish the BMN content. A typical BMN spectrum is shown in fig. 2, together with the absorbance spectrum calculated from $A=-\log \left(I / I_{0}\right)$ for the
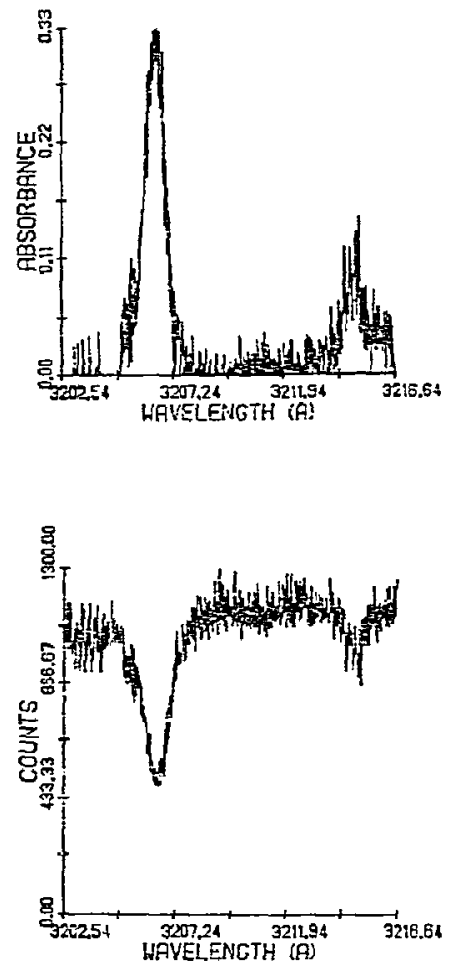

Fig. 2: BMN absorption spectrum of $30 \% \mathrm{C}_{10} \mathrm{H}_{8} / \mathrm{C}_{10} \mathrm{Dg}$. Absorption spsctrum (oottom) of the $30 \%$ guest concertration crystal, $3.4 \times 10^{-4}$ mole fraction $B M N$, at $4.2 \mathrm{~K}$. The BMN $0-0$ band is seen at $3207 \AA$. The absorbance spectrum is also calculated and plotted (top), using the standard relation $A=-\log \left(I / I_{0}\right)$. The integrated intensity of this band is used to determine the composition or the crystal in most cases. Sometines the absorbance peak heights were also used to check for verifration. All calculations however were done consistently crystal having $30 \%$ guest concentration. A standard was established for estimating the molar absorption coefficient, and thus we were able to come to an estimate of the actual BMN concentration in the samples. Table 1 includes the absorption data results, and an average BMN concentration calculated from all the crystals, each one properly weighted according to its $C_{10} D_{8}$ content. As the BMN (perdeuterated) originates from its "natural abundance" in the commercial $C_{10} D_{3}$ sample, its concentration should be proportional to $\left(1-C_{\mathrm{g}}\right)$. Greater confidence can be attributed to the average value, rather than to each individual sample, in view of the difficulties encountered with such low concentrations.

The fluorescence spectra were taken at liquid helium temperatures ( 4.2 and $1.8 \mathrm{~K}$ ) and the emission was monitored for the different components. There is no emission from the $\mathrm{C}_{10} \mathrm{D}_{8}$ species, as has previousiy been established, because it decays rapidly to the lower entegy $\mathrm{C}_{10} \mathrm{H}_{8}$ guest. The " $0-0$ " guest species emission is not strong because it is very weakly allowed and also reabsorbed. We therefore use an intense vibronic band ("0-512") to monitor the guest emission, and compare it with the " $0-0$ " band for the BMN emission. Also, by monitoring a vibronic band, we avoid the problem of reabsorption of emitted radiation in the bulk of the crystal, as would be the case with the " $0-0$ " band of naphthalene (but not the " 0

Table 1

BMN concentrations and averages

\begin{tabular}{lll}
\hline$C_{g}$ & $\begin{array}{l}\text { Concentration from } \\
\text { absorption spectra }\end{array}$ & Average \\
\hline 0.99 & - & $3.4 \times 10^{-6}$ \\
0.95 & - & $1.7 \times 10^{-5}$ \\
0.90 & $3.7 \times 10^{-5}$ & $3.4 \times 10^{-5}$ \\
0.85 & $7.0 \times 10^{-5}$ & $5.1 \times 10^{-5}$ \\
0.80 & $5.6 \times 10^{-5}$ & $6.8 \times 10^{-5}$ \\
0.70 & $7.1 \times 10^{-5}$ & $1.0 \times 10^{-4}$ \\
0.60 & $9.2 \times 10^{-5}$ & $1.4 \times 10^{-4}$ \\
0.50 & $2.6 \times 10^{-5}$ & $1.7 \times 10^{-4}$ \\
0.40 & $1.8 \times 10^{-4}$ & $2.1 \times 10^{-4}$ \\
0.30 & $2.4 \times 10^{-4}$ & $2.4 \times 10^{-4}$ \\
\hline
\end{tabular}


0 " of the BMN, because of the low concentration). Finally, the " $0-512$ " band happens to fall close in energy to the " $0-0$ " of BMN, so it is an ideal comparison band. Fig. 3 shows some of these spectra at $1.8 \mathrm{~K}$. The low energy component is the guest vibronic band, while the high energy one is the supertrap emission. One sees that as $C_{g}$ goes up the supertrap emission increases at the expense of the guest emission in a clear trend, even though the traps absolute (as well as relative to guest) concentration decreases, something contrary to "analytical chemistry" intuition. The same idea holds for fig. 4 where the $4.2 \mathrm{~K}$ spectra are shown.

\section{Percolation and energy transport}

The concentrations of the host, guest, and supertrap species are $C_{\mathrm{h}}, C_{\mathrm{g}}$, and $C_{\mathrm{s}}$, respec-
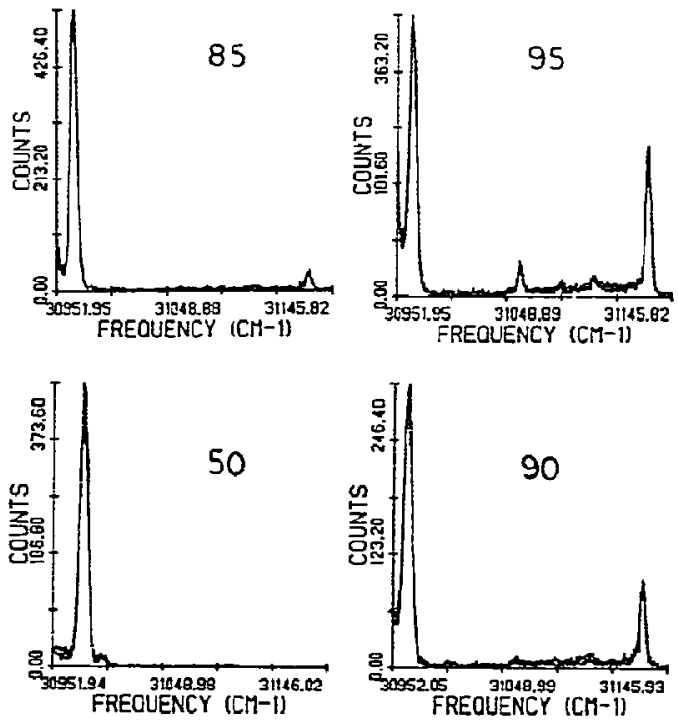

Fig. 3. Fluorescence spectra showing the percolation behavior. Some typical spectra of four crystals at $1.8 \mathrm{~K}$ with concentrations as marked. One can observe the rise of the $B M N$ intensity at the expense of the $\mathrm{C}_{10} \mathrm{H}_{8}$ intensity as the guest concentration goes up. For the complete intensity ratios see table 2. Some minor features that appear in the spectra are attributed to isotopic impurities present in the crystals.
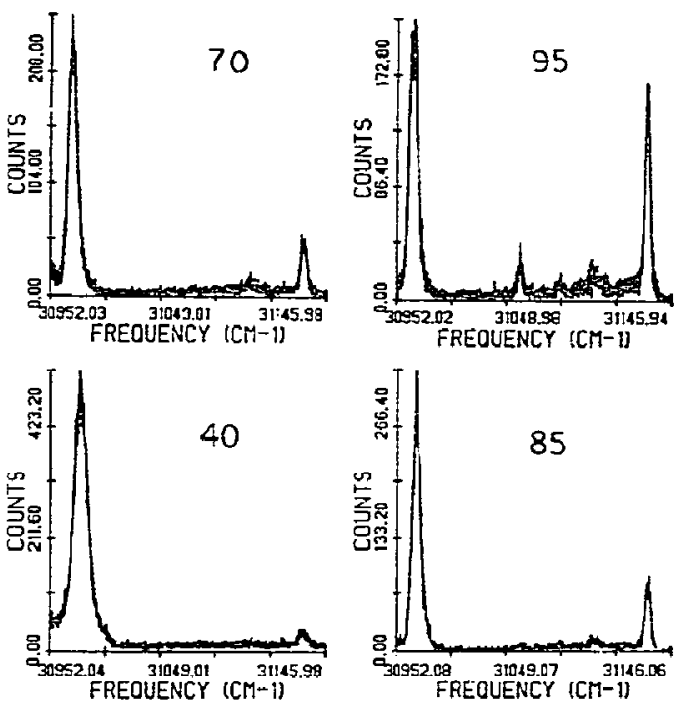

Fig. 4. Fluorescence spectra at 4.2. K. Some typical spectra at $4.2 \mathrm{~K}$ showing the percolation behavior. These spectra are similar to the ones in fig. 3 but at higher temperature.

tively. As mentioned earlier,

$C_{\mathrm{s}} \leqslant 1$.

For a finite crystal it has been shown [31] that the probability of supertrapping $P_{\mathrm{s}}$ is given by:

$P_{\mathrm{s}}=1-\left(1-n_{m} / C_{\mathrm{g}} N\right)^{N C_{\mathrm{s}}}$,

where $N$ is the size of the lattice, $m$ is the size of the largest cluster (maxi-cluster) on which both the transfer and trapping are assumed to occur, and $n_{m}$ is the portion of the lattice in that cluster that has carried excitonic energy. Only in the limit of very efficient transfer can we substitute:

$n_{m}=m$.

This is the case for a long exciton lifetime and/or large exciton interactions and efficient supertrapping, implying that all the available guest sites in the largest cluster have sampled the excitonic energy. Eq. (2) also assumes that supertrapping occurs for every arrival on a trap site, i.e. the supertrapping efficiency is unity. The largest cluster $m$ determines the actual terrain where energy transfer is most efficient 
and measurable. From a topological view it is the percolating cluster and it is first formed at $C_{\mathrm{g}}=0.59$.or a square (two dimensional) lattice, at $C_{\mathrm{g}}=0.31$ for a simple cubic (three dimensional) lattice, etc. Thus, considering a square (two dimensionalj lattice, the usable $C_{\mathrm{g}}$ range is $C_{\mathrm{g}}=0.59-1.00$. For the most general case the trapping probability is:

$P_{\mathrm{s}}=\bar{P}_{\infty}\left[1-\left(1-n_{m} / C_{\mathrm{g}} N\right)^{N C_{\mathrm{s}}}\right]+I_{\mathrm{AV}}^{\prime} C_{\mathrm{s}} / C_{\mathrm{g}}$,

where $\bar{P}_{\infty}$ is the probability of a guest site belonging to the largest cluster, and $I_{A V}^{\prime}$ is the reduced ayerage cluster size [ 44$]$. Here the second term represents the correction to the probability die to the contributions frem smaller clusters.

We use the information we have on $n_{m}, m$, and $\vec{P}_{\infty}$ from the random and correlated walk simulations on binary lattices to estimate the trapping prcbability, $F_{s}$. Experimentally, the relative ratio of the $\mathrm{C}_{10} \mathrm{H}_{8} / \mathrm{BMN}$ (guest-supertrap) intensities is also indicative of the fraction of energy superirapping. Remembering that BMN is about $: 400 \mathrm{~cm}^{-1}$ lower in energy than the $\mathrm{C}_{10} \mathrm{H}_{8}$ guest. it can be seen that if energy is supertrapped by the BMN low lying level. and a radiative dissipative channel is considered, it will eventually emit from this level. Therefore, practically all of the BMN fluorescence indicates excitons that were able to "find" the low concentration component, be trapped by it and. being unable to detrap, eventually iluoresce to the crystai ground electronic state. We define $I$ as the relative trap emission:

$I=I_{\mathrm{s}} /\left(I_{\mathrm{s}}+I_{\mathrm{s}}\right)=I_{\mathrm{s}} / I_{\mathrm{tot}}$,

where $I_{\mathrm{s}}$ is the fluorescence observed from the supertrap, and $I_{\mathfrak{g}}$ that observed from the guest molecules. The quantity $I$ measures the ability of excitonic energy to find the supertraps, during the lifetime of the excitation, and it is equivalent to the calculated quantity $P_{\mathrm{s}}$ which was defined as the probability for supertrapping. Table 2 contains the experimental results of the fraction $I$ in terms of eq. ( 5 ). Since BMN plays the role of the sensor, in this case it is inportant for comparison purposes that it stays constant for all crystals. To do this we normal- ize $I$ to the same $\mathrm{BMN}$ concentration for the whole guest range (last 3 cclumns of table 2). For a large $N$, the term $N C_{g} / n_{m} \rightarrow \infty$ in eq. (2). Also $P_{\mathrm{s}}$ becomes the experimental quantity $I$ (as previously discussed). Therefore, by expanding we have:

$I=\bar{P}_{\propto}\left[1-\exp \left(-C_{\mathrm{s}} n_{m} / C_{\mathrm{g}}\right)\right]$.

For ancther concentration $C_{s}^{\prime}$ there would be a corresponding:

$I^{\prime}=\bar{P}_{\infty}\left[1-\exp \left(-C_{s}^{\prime} n_{m} / C_{\mathrm{g}}\right)\right]$.

Eqs. (6) and (7) are coupled through $n_{m}$ and their simultaneous solution yields:

$I^{\prime} / \bar{P}_{\infty}=1-\left(1-I / \bar{P}_{\infty}\right)^{\prime \prime} / C_{\mathbf{z}}$.

We therefore calculate the intensities $I$ as if all BMN concentrations were the same in the entire $C_{\mathrm{g}}$ range, adjusting them one at a time, using eq. (8). The table 2 entries are plotted in fig. 5 as a function of $C_{y}$. One observes as a result a typical percolation-type behavior. It is seen that while $I$ shows only a "dark current" behavior for $C_{\mathrm{g}} \leqslant 0.70$, it rises dramatically in the region $C_{\mathrm{g}}=0.80$, and is practically 1.0 above this sudden rise, up to the pure crystal point, the sharp shift in behavior occurring between 0.80 and 0.90 . We call this sudden change a form of dynamic percolation. The region below $C_{\mathrm{g}}=0.55$ is the region below the critical (static) percolation concentration $C_{\mathrm{c}}$ (nearest neighbor only interactions), i.e. below the formation of the infinite cluster (square lattice topology). Here the BMN sensor molecules are mostly isolated inside small clusters, which do not communicate with each other, and thus are not accessible to the excitation. We note that the next-nearest-neighbor interactions are toc small to erase energy mismatches among these small clusters (below $C_{\mathrm{c}}$ ). Above $C_{\mathrm{g}}=0.60$ the BMN molecules mostly belong to the largest cluster and thus become more easily accessible, and the result is that a large portion of the excitation is eventually trapped by BMN. The quantitative aspect of trapping depends, of course, on the relative number of BMN sites. This is why variation in the sensor concentration causes a shift in the dynamic percolation curve. 
Table 2

Experimental and normalized fuorescence intensities

\begin{tabular}{|c|c|c|c|c|c|}
\hline \multirow[t]{2}{*}{$c_{\mathrm{g}}$} & \multirow[t]{2}{*}{$I$} & \multirow{2}{*}{$\begin{array}{l}C_{3} \\
\text { actual, } \\
\text { averaged }\end{array}$} & \multicolumn{3}{|l|}{$I$ normalized ${ }^{21}$} \\
\hline & & & $C_{\mathrm{s}}=2.8 \times 10^{-4}$ & $C_{\mathrm{s}}=1 \times 10^{-4}$ & $C_{5}=5 \times 10^{-5}$ \\
\hline 0.99 & 0.10 & $3.4 \times 10^{-6}$ & & & \\
\hline 0.95 & 0.49 & $1.7 \times 10^{-5}$ & $0.999^{\circ}$ & 0.980 & 0.860 \\
\hline 0.90 & 0.38 & $3.4 \times 10^{-5}$ & 0.989 & 0.760 & 0.510 \\
\hline 0.85 & 0.09 & $5.1 \times 10^{-5}$ & 0.440 & 0.190 & 0.100 \\
\hline 0.80 & 0.06 & $6.8 \times 10^{-5}$ & 0.251 & 0.098 & 0.025 \\
\hline 0.70 & 0.03 & $1.0 \times 10^{-4}$ & 0.068 & 0.025 & 0.016 \\
\hline 0.60 & 0.02 & $1.4 \times 10^{-4}$ & 0.038 & 0.014 & 0.009 \\
\hline 0.50 & 0.02 & $1.7 \times 10^{-4}$ & 0.028 & 0.010 & 0.007 \\
\hline 0.40 & 0.02 & $2.1 \times 10^{-4}$ & 0.021 & 0.007 & \\
\hline
\end{tabular}

3) See text.



Fig. 5. Experimental $I=I / I_{\text {rot }}$ versus $C_{g}$ at $1.8 \mathrm{~K}$. The quantities $I$ are plotted as a function of $C_{s}$, normalized according to the discussion, in order to achieve the same supertrap concentration in the whole guest range. The dynamic percolation concentration is a function of the supertrap concentration, and it occurs in the region of about 0.80 for the range of concentrations of our experiments.
A similar behavior is observed when the higher temperature ( $4.2 \mathrm{~K}$ ) data are treated the same way and plotted in fig. 6 . Variation of $C_{5}$ alters this behavior slightly, within the mentioned range, and this dependence can be clearly seen in fig. 5. BMN saturated ternary crystals have been previously studied $[33,34]$, and a BMN concentration of $1 \times 10^{-3}$ yields a "percolation" threshold at about $0.40 \pm_{0} 0.60$ guest fraction. It is seen that as the sensor concentration decreases the dynamic percolation concentration moves to higher $C_{\mathrm{g}}$ values. It is because of this dependence on the sensor concentration that the problem is dynamic in nature, rather than static (where the sensor concentration is not important, when the crystal is saturated with BMN). To understand this behavior one has to look at the results of the static percolation problem as being a limiting case of the more general dynamic percolation problem.

Focusing on naphthalene, it is well known [45] that the strongest interaction occurs along the $\pm \frac{1}{2}(a \pm b)$ direction which is that of the interchange equivalent molecular sites. Therefore, it is reasonable, to a first approximation, to consider a square lattice topology, i.e. each site having four nearest neighbors, all at equal distances and on the same plane. In this case an infinite cluster appears at $C_{\mathrm{g}}=0.593$, and this critical concentration value $C_{c}$ is where the 


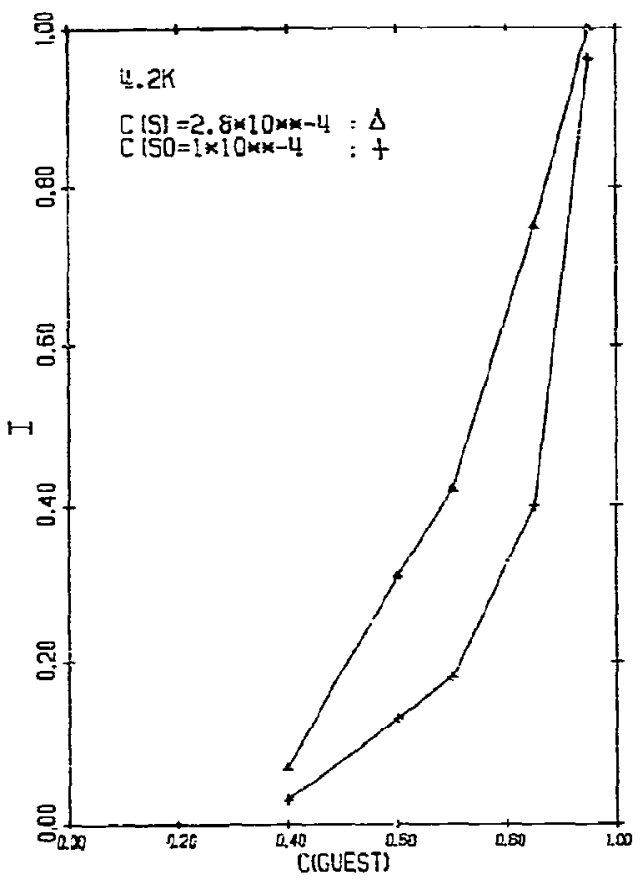

Fig. 6. Experimental $!=I_{5} / I_{\mathrm{zot}}$ versus $C_{\mathrm{g}}$ at $4.2 \mathrm{~K}$. Same as in fig. 4 , but now the temperature is $4.2 K$. See previous figure.

"insulator-to-conductor" shift may first occur. In a real crystal, if the sensor concentration is high enough, then the registration efficiency will parallel the ciuster growth, because in this case the abundance of sensors guarantees that at $C_{\mathrm{g}}=0.593$ where the infinite cluster appears all excitation failing into this cluster will indeed be trapped by some supertrap, and as it was mentioned earlier [33], this "static case" is indeed observed for $C_{s}=1 \times 10^{-3}$.

We focus attention now on the other limit, where the number of sensors present is low, and therefore very critical. Our hasic assumption here is that upon excitation the time element becomes important. The excited state has a well known iifetime, and it is within this lifetime that excitation can reach the sensors. If this does not happen the excitons decay to the ground state from the gliest level, rather than from the supertrap. In effect, there is a competition between the number of sensors and registration efficiency on one hand, and the lifetime of the excited state on the other. The latter parameter is a constant for the system, so we see that the only adjustable quantity for a steady-state experiment is the sensor concentration. The region of interest is above 0.70 guest mole fraction, because as is seen in fig. 3 there the transport and superirapping are relatively efficient within the lifetime. Below this concentration, because of the cluster distribution [31], the finiteness of the lifetime precludes significant energy supertrapping.

It has been shown [36] that the increase of the sensor registration probability with $C_{\mathrm{g}}$ shows a strong dependence on the frequency of phonon scattering as expressed by a parameter describing the coherence length distribution in the pure crystal. An important result was that the long range transport was favored by large coherence values (parameter $l$ ) at high $C_{\mathrm{g}}$, but was hindered at lower $C_{\mathrm{g}}$ (but a $C_{\mathrm{g}}$ which is still well above the critical percolation concentration). We thus invoke the coherence property (l) and search for a value that gives a $C_{\mathrm{g}}$ dependence of transport that best agrees with our experimental resuits assuming that the parameter $l$ is concentration independent (see below).

The registration probability (prosability for trapping) by a sensor is given by [compare eq. (6)]:

$P_{s}=\bar{P}_{\propto}\left[1-\exp \left(-C_{s} \gamma n_{m} / C_{i,}\right)\right]$.

As we discussed earlier the experimental quantity $I$ is a direct measure of $P_{5}$. Then, solving for $\gamma n_{m}$ (the effective number of sites visited) we get:

$\gamma n_{m}=-\left(C_{\mathrm{g}} / C_{s}\right) \log \left(1-I / \vec{P}_{\infty}\right)$.

Table 3 contains these quantities as a function of guest concentration.

The trapping efficiency, $\gamma$, is an important parameter at this point. It is simply a measure of the cross section for trapping. The energy gap in this case is of the order of $400 \mathrm{~cm}^{-1}$, which is an extremely high thermal barrier at liquid helium temperatures. Our hopping mode! 
Table 3

Number of sites visited in simulation and experimental effective number of sites visited

\begin{tabular}{|c|c|c|c|c|c|c|}
\hline \multirow[t]{2}{*}{$c_{\mathrm{g}}$} & \multirow{2}{*}{$\begin{array}{l}\text { Experimental } \\
\gamma n_{m}\end{array}$} & \multicolumn{5}{|l|}{$n_{m}$} \\
\hline & & $l=1$ & $l=10, \mathrm{sd}=3^{a l}$ & $I=25, \mathrm{sd}=3$ & $l=25, \mathrm{sd}=8$ & $t=100, \mathrm{sd}=30$ \\
\hline 0.99 & 33600 & 44710 & 98220 & 107500 & 107350 & 103100 \\
\hline 0.95 & 42700 & 43390 & 92830 & 91390 & 93080 & 82550 \\
\hline 0.90 & 14550 & 40320 & 76440 & 67000 & 74877 & 66270 \\
\hline 0.85 & 1830 & 31120 & 65900 & $4 \cdot 30$ & 59600 & 31970 \\
\hline 0.80 & 820 & 28063 & 40750 & 1190 & 30730 & $1 i 300$ \\
\hline 0.75 & 350 & 22730 & 27230 & & 11848 & 1916 \\
\hline 0.70 & 250 & 15275 & 7219 & & 2478 & \\
\hline $0.6 \mathrm{C}$ & 160 & 3843 & & & & \\
\hline
\end{tabular}

s) $s d=$ standard deviation.

assumes an upper limit for $\gamma$ of the order of 1 , and this is true only when trapping is extremely efficient. Values of $\gamma \gg 1$ have been reported $[3,46]$ in the literature, but experimental evidence [38] seems to be in conflict with these assertions. We also assume here that for BMN $y$ is not a function of $C_{\mathrm{g}}$. All fuorescence spectra, in the entire $C_{\mathrm{g}}$ range, exhibit a BMN band with the same characteristics in all cases (i.e. same frequency and similar phonon side bands), which is at least consistent with this latter assumption (the $\mathrm{BMN}, \mathrm{C}_{10} \mathrm{H}_{8}$ and $\mathrm{C}_{10} \mathrm{D}_{8}$ phonons are all in the amalgamation limit [45]).

The quantities $\gamma n_{m}$ are calculated (table 3 ) using the normalized experimental intensities for the entire $C_{\mathrm{g}}$ range. We then test several coherence models using the $n_{m}$ results reported earlier [37]. We solve for $\gamma$ and plot $\gamma$ versus $C_{\mathrm{g}}$ for $l$ distributions with average values 1,25 , and 100 (gaussian distributions with standard deviation $s d=0,3.0$, and 30.0 , respectively). As it can be seen from table 3 , the cases of $l=10$, sd $=3$, and $l=25, \mathrm{sd}=8$ produce results similar to the $l=100, \mathrm{sd}=30$ case, and, therefore, are not plotted in the same plot, for simplicity. The results are given in fig. 7 . As it is seen from this figure $\gamma$ is not constant for any of the cases. When the distribution of $l$ values is constant (i.e. the standard deviation is about $1 / 3$ the $l$ value) $l=1$ shows a constant increase as $C_{\mathrm{g}}$ increases, while $l=10, l=25, l=100$, show the same trend, although not constant, to a much lesser degree. For example, the greatest variation of $y$ for the $l=100$ case is about a factor of 6 , while the $l=1$ shows a factor of about 50 . For the case of $l=25$ and $\mathrm{sd}=3.0$ this agreement is better than a factor of 2 .

In fig. 8 we plot calculated probabilities for trapping according to the discussion above for the cases of $l=1, l=25(\mathrm{sd}=3.0)$, and $l=25$ (sd =8.0), together with the experimental $I$ results at $1.8 \mathrm{~K}$. Here, the trapping efficiency is taken to be 1 . Again, the $l=25(\mathrm{sd}=3.0)$ case fits rather well, while the $l=1$ case is clearly different. Note that $l=100(\mathrm{sd}=30)$ gives the same result as $l=25(\mathrm{sd}=8)$.

These coherence lengths (expressed in nearest neighbor lattice units) correspond to a minimum coherence time of $10 \mathrm{ps}$, with values such as $50 \mathrm{ps}$ in agreement with this picture $[32,35]$ (using the accepted nearest-neighbor transfer time of about [38] $1 \mathrm{ps}$ ). Energywise this is equivalent [47] to a maximum homogeneous linebroadening of about 0.2 to $1 \mathrm{~cm}^{-1}$.

The coherence length and its distribution are both important factors, and in a way related to each other. The theme of this work is to show that the experimental data suggest explicitly the existence of coherence by showing that the $l=1$ case does not fit the data. However, these data cannot lead to any refined decisions about the exact value of $l$ and its distribution.

The relation between the coherence $l$ and the distribution that its values are likely to have has 




Fig. 7. Trapping efficiency as a function of $C_{5}$. The trapping efficiencies are plotted as a function of $C_{\mathrm{s}}$ tor different types of walks. Ore is iooking for a behavior that stays more or less constant in the total range of the guest concentration. The efficiency of the randon walk in each cuse determines the derived trapping efficiency according to the formalism of eq. (9). The cases of $l=10$. sd $=3$. and $l=25$, $\mathrm{sd}=8$ produce results similar to the $l=100$, sd $=30$ case and are omitted from this plot for simplicity. Here the triangle refers to the case $l=1$, the star to the case $l=25(\mathrm{sd}=3.0)$, and the cross to $I=100$ (with $s d=30.0$ ).

also been of interest [48] from a difierent point of view. Simple criteria can be set that establish the connection. For example, for an esiablished number of $l$ correlated steps, the equation $y=$ $\exp (-x / l)$ describes the probability at any instance that a number of $x$ (where $x$ is a variable inieger) correlated steps is followed. The relation between $x$ and $l$ can be set arbitrarily; for example, establisin such a connection by imposing that the area under the $y=\exp (-x / l)$ curve from $(l-x)$ to $(l+x)$ is equal to the remaining area, i.e., the portion from 0 to $x$ plus the portion from $(1+x)$ to infinity. This

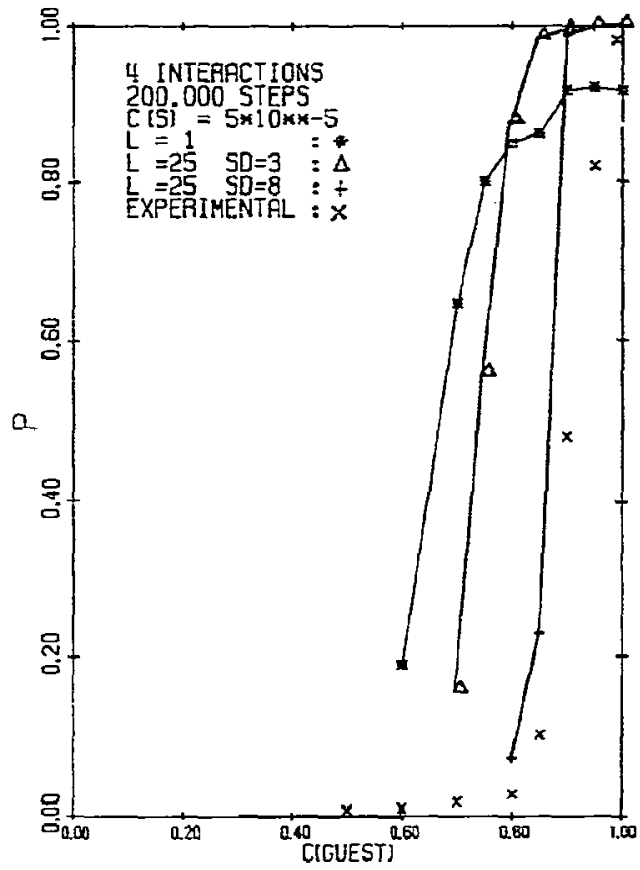

Fig. 8. Registration probability is a function of $C_{\mathrm{g}}$. The registration probabilities are plotted as a function of $C_{b}$ according to the discussion of section 3 . Several different walks are employed as shown in the figure. The experimental intensities are also plotted on the same scale for comparison. One can see that the actual behavior itrongly depends on the parameters of the random walk. and in particular on the coherence, $l$, and its standard deviation, sd. The case of $l=100 . \mathrm{sd}=30$ produces resulis at this point that are very close to the case $l=25, \mathrm{sd}=\mathrm{S}$. The temperature here is $1.8 \mathrm{~K}$, and the trapping efficiency is taken for convenience to be 1 .

condition results in $l / x=1.6$. Of course, different criteria could be set resulting in a different $l / x$ ratio. For some of our calculations we have used a ratio of about 3 , which is within a factor of 2 if the above condition is obeyed.

The effect of topology has also been previously discussed. The general conclusion is that the larger the coordination number (the number of bonds), the higher the visitation efficiency (and the calculated quantity $P$ ). This effect is shown in figs. 2,4 and 5 of our recent paper [36], where the number of bonds grows from 4 to 6 to 8 . This shows that if we include any 
interactions in addition to the four nearest neighbor ones the dynamic percolation curve shifts to the left (from that in fig. 7), and gives a greater discrepancy with the experimental results. This is reasonable because it has previously been shown [49] that the naphthalene system is nearly a 2-dimensional system in regard to energy transfer, and the non-nearest neighbor interactions are smaller. The fac: that the latter interactions are of drastically reduced relevance near $C_{\mathrm{c}}$ has been shown by Kopelman et al. [34], and is caused by an energy mismatch which has been neglected in our simulations here (see below).

The coherence times derived above should be mostly due to exciton-phonon scattering because effects due to impurity and/or imperfection scattering should be negligible in systems where $10-40 \%$ of the sites are already built-in scatterers ("host"). We emphasize here that the host scattering is explicitly accounted for in the computer simulation $[2,36]$ and does not affect the parameter $l$, and the "scattering time" derived from it. The latter two pertain to pure guest (or host) systems. We thus assume that our scattering time represents the excitonphonon scattering time ${ }^{*}$. On the other hand, usually ordinary spectroscopic linebroadening measurements only give upper limits to the exciton-phonon (homogeneous) linebroadening because of the always possible contributions from inhomogeneous linebroadening, owing to lattice imperfections. Also part of the homogeneous broadening is due to other factors (e.g.,

\footnotetext{
*We assume that the exciton-phonon coupling is the same for pure $\mathrm{C}_{10} \mathrm{H}_{8}$ and for mixed $\mathrm{C}_{10} \mathrm{H}_{8} / \mathrm{C}_{10} \mathrm{D}_{8}$ crystals. The phonons of $\mathrm{C}_{10} \mathrm{H}_{4}$ and $\mathrm{C}_{10} \mathrm{D}_{4}$ are in the amalgamation limit [45] throughout the whole concentration range, as has been amply demonstrated from Raman and farinfrared studies [66]. The minute amounts of BMN in our sample do not change this situation because: (1) BMN and naphthalene are also [65] in the amalgamation limit. (2) The amounts of BMN in these samples are less than in most "pure" naphthalene samples. (3) Studies of the BMN phonon sideband [67] have shown no localized phonons and no changes with guest concentration $\left(\mathrm{C}_{10} \mathrm{H}_{8} / \mathrm{C}_{10} \mathrm{D}_{8}\right)$. As to notions that in a pure $\mathrm{C}_{10} \mathrm{H}_{8}$ cry'stal the wavevector conservation effects (due to translational symmetry) will cause difierent phonon scatiering
}

presence of C-13, etc.) $[7,45,61]$. However, we note that the narrowest linewidths measured for this system, on carefully prepared samples. have been [62] about $0.3 \mathrm{~cm}^{-1}$, and the extrapolated phonon-caused homogeneous linebroadening is an order of magnitude smaller [50]. Thus, there appears to be good agreement between the lower limit to the first singlet exciton coherence time derived from spectral linewidths and that derived here from the exciton percolation method [32] (see discussion). On the other hand, we emphasize that our "coherence length" is but a measure of "hopping correlation", as discussed by us in terms of a correlated-hopping-nodel $[32,37]$.

We expand somewhat on our model. We believe that "correlated hopping" can describe the situation between the limit of "incoherence" (hopping model) and the limit of "coherence" (band model). While in small clusters (dimers) it is casy to talk about "coherence", the situation in a pure crystal may be different [68]. We believe that the model of "correlated hopping" can be applied to both "neat" crystals (at finite temperatures) and to mixed crystals (high $C_{\mathrm{g}}$ ). It is an alternative to the use of the band medel for both neat and mixed (high $C_{\mathrm{g}}$ ) crystals. Thus the correlated hopping enables us, at least in principle, to separate impurity scattering from phonon scattering in mixed (high $C_{\mathrm{g}}$ ) crystals. We note that usually "coherence" in this field means band motion, i.e. $D \approx\left\langle v_{k}^{2} \tau_{k}\right\rangle \approx\left\langle l_{k}^{2} \tau_{k}^{-1}\right\rangle$, with $v_{k}, l_{k}$ and $\tau_{k}$ the exciton $k$ states velocity, free path and (phonon) scattering time, while

\author{
than in the ternary crystal, we remind the reader that \\ "pure" $\mathrm{C}_{10} \mathrm{H}_{8}$ always contuins about $11 \%$ mole of \\ monocarbon-13-naphthalene $\left({ }^{13} \mathrm{C}^{12} \mathrm{C}_{4} \mathrm{H}_{\gamma}\right)$, and usually \\ contains 10-100 times more BMN than our samples. In \\ addition. "pure" $\mathrm{C}_{10} \mathrm{D}_{8}$ usually contains about $10 \%$ mole \\ of $\mathrm{C}_{10} \mathrm{D}_{7} \mathrm{H}$ as well as $11 \%{ }^{13} \mathrm{C}^{13} \mathrm{C}_{9} \mathrm{D}_{8}$ and $10-100$ times \\ more BMIN than our samples. Thus we believe that our \\ ternary crystals have as much effective translational \\ symmetry (as far as the phonons are concerred) as so- \\ called pure naphthalene crystals and thus have effectively \\ the same exciton-phonon interactions and scattering. \\ Therefore, our parameter $l$ should be valid for pure, per- \\ fect naphthalene as well as for our experimentally studied \\ samples.
}


incoherence means hopping, i.e. $D=W$, where $W$ is the (ilearest neignbor) hopping rate, which in the Holstein model is $W \approx \beta^{2} \omega_{0}^{-1}$ with $\beta$ the nearest neighbor matrix element and $\omega_{0}$ a vibrational frequency. At high $C_{b}$, at these low temperatures, the exciton band states may be close to being eigenstates so that a band picture with coherence in the above range may be almost correct. However, the band model, which originally implied totally coherent Bloch states, has ofter been pushed to the extreme of an $l_{k}$ approaching one latice unit, which is essentially the incoherent hopping limit. Here we start from the opposite limit of incoherent hopping $l=1$ (meaning $l_{k}=$ one lattice unit), and progress towards the limit of full coherence via the device of "hopping correlation", which enables a simple computer simulation of the semicoherent casc with an intermediate $l_{k}$. This model has the advantage of easy adaptation to binary lattices (well above $C_{\mathrm{c}}$ ). The relative merits of this and other models will have to face further tests. Conceptually our model gives the particle a partial directional memory, in some analogy to memory functions [53].

\section{Temperature efiects}

Fig. 6 shows our experimental results for $4.2 \mathrm{~K}$ temperature. These data were analyzed in a similar fashicn to the $1.8 \mathrm{~K}$ case, and they were recorded on the same samples, usually on the same day. At this stage this effect will be treated only qualitatively. We observe that $I$ is greater in all cases at $4.2 \mathrm{~K}$ than at $1.8 \mathrm{~K}$. All theoretical incolels $[5,32,51]$ predict that a continuous change of the character of motion occurs from purely coherent to purely incoherent with increasing temperature. The increased exciton-phonon coupling causes now more scattering evenis per unit time, which effectively do not allow $I$ to "build-up" to higher values. This results in smaller coherence lengths at higher temperatures. Small coherence lengths, throughout most of the guest region (except when approaching the neat crystal limit), have a higher visitation efficiency, which in turn increases the probability of trapping by the supertrap. Therefore, $I$ should indeed inctease at $4.2 \mathrm{~K}$. The situation, however, may be more complex.

In general, we have "phonon-assisted-correlated hopping". In particular, the cluster distribution in these binary systems, at certain concentrations, produces a very complex terrain of sizes and shapes. In principle, all molecular energy levels have the same energy, but in these binary systems an energy mismatch occurs because of the cluster characteristics. We performed some simple calculations on small size clusters, and found that indeed this is the case. This results in a variety of shallow traps, where the temperature effect will be mostly pronounced. At the higher temperature detrapping can occur, which further increases the experimental quantity $I$. This efiect may be pronounced between $C_{\mathrm{c}}(0.593)$ and about $C_{\mathrm{g}}=$ 0.70 . It is most important at or below $C_{e}$, as discussed elsewhere [63]. However, it should not affect much the transport at high $C_{\mathrm{g}}$, our main region of interest.

Let us focus now on the high guest concentrations, say above $C_{\mathrm{g}}=0.95$. Here one expects that the high $l$ walk is more efficient than $l=1$. Therefore, the low temperature spectra should show a higher $I$. However, as the temperature increases to $4.2 \mathrm{~K}$ the coherence length may be reduced, but not quite down to total incoherence. This means that, for example, if $t=$ 1000 at $1.8 \mathrm{~K}$ and $l=100$ at $4.2 \mathrm{~K}$ we do not expect any differences. Within our model, as it was mentioned earlier, the cases $l=100$ and $l=$ 1000 show pretty much the same behavior in this region, su our test is still too rough. One would have to get very close to $C_{g}=1.0$, i.e. meticulously test the region of 0.98-0.995 mole guest fraction, at several temperatures, before any distinction between these $l$ values could be made. For instance, at 0.995 , an $l=10$ and an $l=100$ case would show different behavior, which quantitatively would determine the proper value at each temperature. This project is reserved for the future.

A more extended temperature range, perhaps to boiling nitrogen or room temperatures, could 
be informative in principle. However, our system, because of the fixed energy gaps, would not be proper. One needs to choose a different trap instead of the BMN, that lies lower in energy to produce a deeper energy gap. At the higher temperatures the problem of energy detrapping from the supertrap back to the guest level may complicate the calculations of intensity ratios. First, we estimate the probability for this to happen: At room temperature for the $400 \mathrm{~cm}^{-1}$ gap and an average supertrap concentration of $C_{5}=1 \times 10^{-4}$ the fraction will be $\left(C_{\mathrm{g}} / C_{\mathrm{s}}\right) \exp [-E$ (gap) $/ k T]$, which is about 1500 , while at $77 \mathrm{~K}$ it is about 5 . This is why a molecule with different energy level must be chosen for stch a study. Another important question is how good a scatterer will $\mathrm{C}_{10} \mathrm{D}_{8}$ be when it can be easily excited by a thermalization of the $\mathrm{C}_{10} \mathrm{H}_{8}$ exciton. Certainly the exciton percolative transport (avoiding host sites) will approach a diffusive form of transport at high temperature.

We conclude that the higher temperature experiments $(4.2 \mathrm{~K}$ ) agree qualitatively with the general picture o: coherence we presented earlier, but the region investigated does not offer any further limiting suggestions on the degree of coherence or hopping correlation (l).

\section{Discussion}

To our knowledge, this is the first study in which the problem of exciton "coherence" in singlet states is quantitatively attacked without the utilization of temperature effects. This method owes its basis to the development of the percolation approach. We utilize the fact that the correlated long hops behave differently from the short ones when they take place in the labyrinths of the clusters in the lattice. This behavior is the opposite from when there is an "open field", as in a perfect lattice. Because of the system complexities it is very difficult to describe the picture without using percolation theory.

The idea of "coherence length" as a mean free path is an old one. Holstein [52] has given a quantum mechanical formalism based on diatomic lattices where the exiton-phonen interaction is the important parameter. In his theory he describes the energy transfe: as a series of tunnelings of the polaron (having a certain coherence length). Munn and Silbey $[5,13,64]$ have reviewed the theoretical formalism concentrating on the exciton-phonon coupling. The development has many similarities to the polaron theory. The resulting solution shows that the transfer of electronic excitation has a wave-like (coherent) part and an incoherent (diffusive) part. To compare with the experiment, the spectral line shapes are calculated (for the absorption) and given in terms of the integral of the correlation function of the dipole moment. To a first approximation, this results in a lorenzian line shape. Thus, the description of exciton migration is given here in a classical model, where the exciton-phonon interactions are replaced by random processes. Finally, the quantum mechanical effects are also included in a further refinement of the theory by adding to the hamiltonian the molecular vibrations of each molecule, and solving the equations of motion once again. Kenkre and Knox [53] have produced a formalism arriving at the same resuits as Silbey, but using a different method. They derive a generalized master equation for the diagonal density matrix elements using projection operator techniques.

The triplet state case has been a subject of greater discussion than the singlet state case, and, in principle, coherence is an exciton property that could be subjected to the same treatment in both cases (taking into account the differences in dynamics of the two states). Harris et al. $[54,560]$ have performed ESR experiments in the triplet state of 1,2,4,5-tetrachlorobenzene, a well known pseudo-1-dimensional system, and have arrived at very precise values of a minimum coherence time of $2.6 \times$ $10^{-9} \mathrm{~s}$, and a minimum coherence length of $700 \AA$. This involves a value $N=2.56 \times 10^{5}$, where $N$ is the number of molecules in the linear chain (or the average number of exciton states pér trap). However, a different interpretation of these experiments, and evidence 
from optical spectra and CDMR spectra as explained by Davidovich [57], has indicated that incoherent motion is not inconsistent with the experimental evidence presented up to now. This definitely throws some doubts on the very precise values reported by Harris et al. $[54,56]$. However, Harris and Zwemer [6] conclude in their review paper that $l$ is several orders of magnitude larget than unity, at least in some systems (tetrachlorobenzene, see also Diott et al. [58] and especially Wieting and Fayer [30]).

Returning to the singlet system of naphthalene, we again deal with the dangerous exercise (see Harris and Zwemer [6], and Francis and Kopelman [32]) of comparing results with optical linewidth studies. The most recent value for the naphthalene singlet exciton is [50] $0.03 \mathrm{~cm}^{-1}$ at $1.8 \mathrm{~K}$. Using a simple uncertainty-principle type approach $[32,38]$ we get about $l=10^{3}$. This is consistent with our result $\left(l \geqslant 10^{2}\right)$ if we assume that in the pure crystal the major contribution to the homogeneous linewidth is due to exciton-phonon scattering (see reservation above and in Francis and Kopelman [32]). Furthermore, we assumed that this phonon scattering is the same for pure $\mathrm{C}_{10} \mathrm{H}_{8}$ and mixed $\mathrm{C}_{10} \mathrm{D}_{\mathrm{S}} / \mathrm{C}_{10} \mathrm{H}_{8} / \mathrm{BMN}$ crystals, as the phonons of all three components are amalgamated $[45,65]$. This was the justification for our tentative conclusion that the $l$ values should be constant throughout our concertration range and applicable to the gure naphthalene crystal. We emphasize again that our "coherence" values $l$ apply only to exciton-phonon scattering and not to the scattering by the host $\left(C_{10} D_{3}\right)$ sites. The latter scattering is explicitly accounted for in the computer simulation of the correlated random walk $[36,37]$. Finally we note that our results here are consistent with the results [59] from our time resolved experiments [60] (paper M).

In conclusion energy transport in these systems is consistent with a kinetic model (dynamic percolation), with correlated hopping limited to the percolating cluster and a mean free exciton path, extrapolated to the pure crystal, equal or larger than 100 lattice units. Our correlated-hopping model is essertially equivalent to a band mocel at high $C_{g}\left(C_{\varepsilon} \rightarrow 1\right)$ and high $/(>100)$. At this limit one indeed expects a temperature increase to result in an exciton transport decrease. At the crossover concentration $[36,37]\left(C_{g}=C_{x}=0.85 \pm 0.1\right)$ this temperature effect reverses itself, as now lower $l$ values increase the transport. Thus one effectively gets a hopping-like behavior, say at $C_{\mathrm{g}}=0.75$, for the same l value that gives a bandlike behavior for the pure crystal $\left(C_{\mathrm{g}} \rightarrow 1\right)$. The model thus automatically converts a negative temperature effect for a pure crystal into a positive temperature effect for a highly doped crystal. However, it should be noted that additional temperature assistance effects may become important as the increased doping reduces the donor concentration $\left(C_{\mathrm{g}}\right)$ towaris the percolation concentration $\left(C_{\mathrm{c}}\right)$. These are phonon-assistance effects due to energy mismatches between various clusters, as mentioned above and discussed before $[32,63]$. However, the importance of these effects is expected to diminish progressively with $C_{\mathrm{g}}$, as the percolating (infinite) cluster annexes all small clusters and becomes the dominant donor (guest) feature, eventually approaching a pure donor crystal. In summary, this paper (1) neglects the thermally assisted exciton hopping (this point is under further study) and (2) invokes a picture according to which exciton scattering by phonons reduces transport, exciton scattering by host sites (antitraps) reduces transpott as well, but where a combination of host scattering and phonon scattering may increase transport, relative to the same host scattering with no phonon scattering. The experimental results appear to be consistent with this model, but further studies are necessary to test its physical reality.

\section{References}

[1] C.E. Swenberg and N.E. Geacintov, in: Organic molecular photophysics, Vol. 1, ed. J.B. Birks (Wiley, New York, 1973);

N.E. Geacintov and C.E. Swenberg, in: Luminescence spectroseopy, ed. M.D. Lumb (Academic Press, New York, 1978).

[2] R. Kopelman, in: Topics in applied Physics, Vol. 15, ed. F.K. Fong (Springer, Berlin, 1976) p. 297. 
[3] R.C. Powell and Z.G. Soos, J. Lumin. 11 (1975) 1.

[4] H.C. Wolf and II. Port. J. Lumin. 12/13 (1976) 33.

[5] R. Sibcy, Ann. Rev. Phys. Chem. 27 (1976) 203.

[6] C.B. Harris and D.A. Zwemer, Ann. Rev. Phys. Chem. $29(1978) 473$.

[7] D.M. Burland and A.H. Zewail, Advances in chemical physics, Vol. 40, eds. I. Prigogine and S.A. Rice (Wiley, New York, 1979).

[8] A. Blumen, J. Klafter and R. Silbey, J. Chem. Phys, 72 (1980) 5320 .

[9] J. Klafter and R. Silbey, J. Chem. Phys. 72 (1980) 843.

[10] J. Klafter and R. Silney, J. Chem. Phys. 72 (1980) 849.

[11] K. Allinger and A. Blumen, J. Chem. Phys. 72 (1980) 4608.

[12] J. Klafter and R. Silbey, Phys, Rev. Letters 44 (1980) 55.

[13] R.W. Munn and R. Silbey, J. Chem. Phys. 68 (1978) 2439 .

[14] A. Blumen and R. Silbey, J. Chem. Phys. 70 (1979) 3707.

[15] R. Silbey and R.W. Mınn, J. Chem. Phys. 72 (1980) 2763.

[16] A. Blumen, J. Chem. Phys. 72 (1980) 2632.

[17] K. Godzik and J. Jortrer, J. Chem. Phys. 72 (1980) 4471 .

[18] D.E. Cooper, R.W. Olson and M.D. Fayer, J. Chem. Phys. 72 (1980) 2332.

[19] K.A. Nelson and M.D. Fayer, J. Chem. Phys. 72 (1980) 5202.

[20] J.P. Lemaistre and A.H. Zewail, J. Chem. Phys. 72 (1980) 1055 .

[21] D.D. Smith, D.P. Millar and A.H. Zewail, J. Chem. Phys. 72 \{1980\} 1187.

[22] A.H. Zewail, J. Chem. Phys. 70 (1979) 5759.

[23] H. Vries and D.A. Wiersma. J. Chem. Phys. 72 (1980) 1851.

[24] L. Altwegg and I. Zschokke-Granacher, J. Chem. Phys. 73 (1980) 213.

[25] S.E. Webter and C.E. Swenberg, Chem. Phys. 49 (1980) 231

[26] R.A. Auerbach, G.W. Robinson and R.W. Zwanzig, J. Chem. Phys. 72 (1980) 3528.

[7] I.I. Abram and R.M. Hochstrasser, J. Chem. Phys. 72 (1980) 3617.

[28] X. Funabashi, J. Chem. Phys. 72 (1980) 3123.

[29] W.H. Hesselink and D.A. Wiersma, J. Chem. Phys. 73 (1980) 648.

[30] R.D. Wieting and M.D. Fayer, J. Chem. Phys. 73 (1980) $7 \div 4$.

[31] J. Hoshen and R. Kopelman, J. Chem. Phys. 65 (1976) 2817.

[32] A.H. Francis and R. Kopelman, in: Topies in applied physics, eds. W.M. Yen and P.M. Selzer (Springer, Berlin, 1981).

[33] R. Kopelman, E.M. Monberg and F.W. Ochs, Chem. phys. 21 (1977) 373.
[34] R. Kopelman, E.M. Monberg and F,W, Ochs, Chem. Phys. $19(1977) 413$.

[35] P. Argyrakis and R. Kopelman, J. Chem. Phys. 66 (1977) 3301.

[36] P. Argyrakis and R. Kopelman, J. Theor. Biol. 73 (1978) 205.

[37] P. Argyrakis and R. Kopeman, Phys. Rev. B 22 (1980) 1830.

[38] P. Argyrakis and R. Kopelman, Chem. Phys. 51 (1980) 9.

[39] M. Kasha, J. Opt. Soc. Am. 38 (1949) 929.

[40] F. W. Ochs and R. Kopalman, App. Speciry. 30 (1976) 306.

[41] P. Argyrakis, Doctozal Dissertation. University of Michigan (1978).

[42] J. Hoshen and R. Kopelman. Phys. Rev. E 14 (1976) 3438.

[43] D.C. Ahlgren, E.M. Monberg and R. Kopelman, Chem. Phys. Letters 64 (1979) 122.

[44] J. Hoshen, R. Kopelman and E.M. Monberg, J. Stat. Phys. 19 (1978) 219.

[45] R. Kopelman, in: Excited states, Voi 2, ed. E.C. Lim (Academic Press, New York, 1975).

[46] H. Auweter, Doctoral Dissertation, University of Sturigart (1978).

[47] J.B. Birks, Organic molecular photophysics, Vol 2 (Wiley, New York 1975).

[48] E.W. Montroll, private comntunication.

[49] H-K. Hong and R. Kopelman, J. Chem. Phys. 55 (1971) 724.

[50] G.J. Small, private communication (1979).

[51] E. Schwarzer, Z. Physik B 20 (1975) 185.

[52] T. Holsiein, Ann. Phys. 8 (1959) 325; 8 (1959) 343.

[53] V.M. Kenikre and R. Krox, Phys. Rev. B 9 (1974) 5279; Phys. Rev, Letters 33 (1974) 803.

[54] R.H. Shelby, A.H. Zewail and C.B. Harris, J. Chem. Phys. 64 (1976) 3192.

[55] A.H. Francis and C.B. Harris, Chem. Phys. Letters 9 (1971) 188.

[56] M.D. Fayer and C.B. Harris, Phys. Rev. B 9 (1975) 748 .

[57] M. Davidovich, Doctoral Dissertation, University of Rochester (1976).

[58] D.D. Dlot, M.D. Fayer and R.D. Wieting. J. Chem. Phys. 67 (1977) 3808;

R.D. Wieting, M.D. Fayer and D.D. Dlot, J. Chem. Phys. 69 (1978) 1996.

[59] R. Kopelman and P. Argyrakis, J. Chem. Phys. 72 (1980) 3053 .

[60] P. Argyrakis and R. Kopelnuan, in preparation.

[61] J.C. Iaufer and R. Kopelman, J. Chem. Phys. 57 (1972) 3202 .

[62] S.L. Robinette, S.H. Stevenson and G.J. Small, J. Chem. Phys. 69 (1978) 5231.

[63] R. Kopelman and E.M. Monberg. Mol. Cryst. Liquid Cryst. 57 (1980) 271. 
[64] R. Silbey and R.W. Munn, Org. Coatings Plastic Chem. 42 (1989) 364.

[65] R. Kopelman, J. Phys. Chem. 80 (1976) 2191.

[66] P.N. Prasad and R. Kopelman, J. Chem. Phys. ST (1972) 863;
D.C. Ahlgren and R. Kopelman, Chem. Phys. 48 ' (1980) 47.

[67] S. Gentry and R. Kopelman, unpublished.

[68] I. Abram, IXth Molecular Cystal Symposium, Mittelberg. Kleinwalsertal (1980) p. 11. 